\title{
Spectra of the International Geomagnetic Reference Field and Its Secular Variation ${ }^{\dagger}$
}

\author{
J. A. OstrowsKi* \\ Division of Seismology and Geomagnetism, Earth Physics Branch, \\ Energy, Mines and Resources, Ottawa, Ontario, Canada
}

(Received April 14, 1982)

The Fourth Scientific Assembly of IAGA adopted a new set of International Geomagnetic Reference Field models for the period 1965.0-1985.0 (IAGA Division I WORKING Group 1, 1981). The set consists of the three "definitive" main field (MF) models for the epochs 1965.0, 1970.0, 1975.0 and of the "international" MF model for 1980.0, along with the predictive secular variation (SV) model for the interval 1980-85. The MF models are of degree/order ten, the SV model is of degree/order eight. Since the new scheme specifies a linear interpolation between MF models for the intermediate epochs, the set of the SV models for the intervals $1965-70,1970-75$, and 1975-80 can be obtained from the differences of the MF models. There are discontinuities between the SV models at the epochs 1970.0, 1975.0, and 1980.0.

The purpose of the present note is to show the behaviour of the Earth's magnetic field in the interval 1965-1985, as described by the set of the new International Geomagnetic Reference Field models. The quantities used are the spectral amplitudes of the field models.

The amplitude $A_{n}(T)$ of the $n$ th-degree MF harmonic at the epoch $T$ is defined as a root square magnitude of the harmonic, taken over the entire surface of the Earth. According to OsTROWSKI (1982) it is given by

$$
A_{n}(T)=\sqrt{(n+1) \sum_{m=0}^{n}\left[\left(g_{n}^{m}\right)^{2}+\left(h_{n}^{m}\right)^{2}\right]} .
$$

The amplitudes of the total field $\left(A_{\mathrm{TF}}(T)\right)$, non-dipole field $\left(A_{\mathrm{ND}}(T)\right)$ and non-quadrupole field $\left(A_{\mathrm{NQ}}(T)\right)$ are obtained from the amplitudes of the individual harmonics:

$$
\begin{aligned}
& A_{\mathrm{TF}}(T)=\sqrt{\sum_{n=1}^{n^{*}}\left(A_{n}(T)\right)^{2}}, \\
& A_{\mathrm{ND}}(T)=\sqrt{\sum_{n=2}^{n^{*}}\left(A_{n}(T)\right)^{2}},
\end{aligned}
$$

${ }^{\dagger}$ Contribution from Earth Physics Branch No. 1023.

*On leave from the Institute of Geophysics, Polish Academy of Sciences, Warsaw, Poland. 


$$
A_{\mathrm{NQ}}(T)=\sqrt{\sum_{n=3}^{n^{*}}\left(A_{n}(T)\right)^{2}},
$$

with $n^{*}$ representing the maximum degree/order of the analyzed model. In the case of the secular variation model, the coefficients $\dot{g}_{n}^{m}, \dot{h}_{n}^{m}$ are used instead of $g_{n}^{m}, h_{n}^{m}$ and the epoch $T$ is replaced by the five-year interval to which the secular variation model applies.

For reference, Table 1 shows the amplitudes of the main field models and Table 2 shows the amplitudes of the secular variation models. The results are also plotted in two different ways and discussed briefly in the following.

In Fig. 1, the MF amplitudes of total field, non-dipole field, non-quadrupole field and of the individual harmonics are plotted as functions of time. The values for the epoch 1985.0 are obtained by applying the IGRF 1980 secular variation model to the IGRF 1980 main field model. Since the IGRF SV model for 1980-85 does not have the ninth and the tenth terms, the values of the MF ninth and tenth harmonics are constant in the interval 1980-85. The amplitudes for the SV models are shown in Fig. 1 as horizontal bars, each covering the five-year interval of the validity of the given SV model.

The plots for the main field are very smooth-almost all of them can be closely represented by a linear or quadratic function of time. The behaviour of the total field is dominated by the linear decay of the first-degree harmonic amplitude. The non-dipole field amplitude, on the other hand, consistently increases due to the increase of the amplitudes of all but the fourth and the sixth-degree harmonics. The non-quardrupole field amplitude also increases, although at a much slower rate. Comparison of the first and the second row of Fig. 1 shows that the behaviour of the total, non-dipole and non-quadrupole fields is determined by the lowest-degree harmonic included in each of these fields.

Table 1. Amplitudes of the geomagnetic reference field models.

\begin{tabular}{cccccccccccccc}
\hline Epoch & $A_{\text {TF }}$ & $A_{\text {ND }}$ & $A_{\text {NQ }}$ & $A_{1}$ & $A_{2}$ & $A_{3}$ & $A_{4}$ & $A_{5}$ & $A_{6}$ & $A_{7}$ & $A_{8}$ & $A_{9}$ & $A_{10}$ \\
\hline 1965.0 & 44929 & 10128 & 6891 & 43772 & 7422 & 5826 & 3297 & 1383 & 776 & 355 & 135 & 122 & 46 \\
1970.0 & 44784 & 10233 & 6916 & 43599 & 7542 & 5865 & 3276 & 1405 & 750 & 360 & 132 & 119 & 45 \\
1975.0 & 44627 & 10347 & 6943 & 43411 & 7672 & 5910 & 3251 & 1419 & 730 & 365 & 133 & 120 & 45 \\
1980.0 & 44488 & 10495 & 6977 & 43233 & 7841 & 5967 & 3216 & 1426 & 716 & 379 & 144 & 124 & 48 \\
1985.0 & 44349 & 10648 & 7008 & 43052 & 8016 & 6024 & 3177 & 1425 & 717 & 381 & 164 & 124 & 48 \\
\hline
\end{tabular}

All values in nanotesla.

Table 2. Amplitudes of the geomagnetic reference field secular variation.

\begin{tabular}{cccccccccccccc}
\hline $\begin{array}{c}\text { Time } \\
\text { interval }\end{array}$ & $A_{\mathrm{TF}}$ & $A_{\mathrm{ND}}$ & $A_{\mathrm{NQ}}$ & $A_{1}$ & $A_{2}$ & $A_{3}$ & $A_{4}$ & $A_{5}$ & $A_{6}$ & $A_{7}$ & $A_{8}$ & $A_{9}$ & $A_{10}$ \\
\hline $1965-1970$ & 75.9 & 66.2 & 39.8 & 37.0 & 52.9 & 30.9 & 16.4 & 12.2 & 11.1 & 7.1 & 5.0 & 2.4 & 3.3 \\
$1970-1975$ & 78.9 & 67.2 & 40.1 & 41.2 & 53.9 & 29.0 & 19.5 & 13.1 & 12.0 & 6.7 & 5.0 & 1.5 & 1.3 \\
$1975-1980$ & 82.0 & 71.3 & 36.6 & 40.4 & 61.2 & 19.6 & 24.6 & 11.1 & 11.0 & 6.6 & 6.1 & 4.3 & 2.7 \\
$1980-1985$ & 82.8 & 71.3 & 39.0 & 42.0 & 59.8 & 21.3 & 25.7 & 12.3 & 11.3 & 8.2 & 7.7 & - & - \\
\hline
\end{tabular}

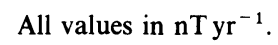



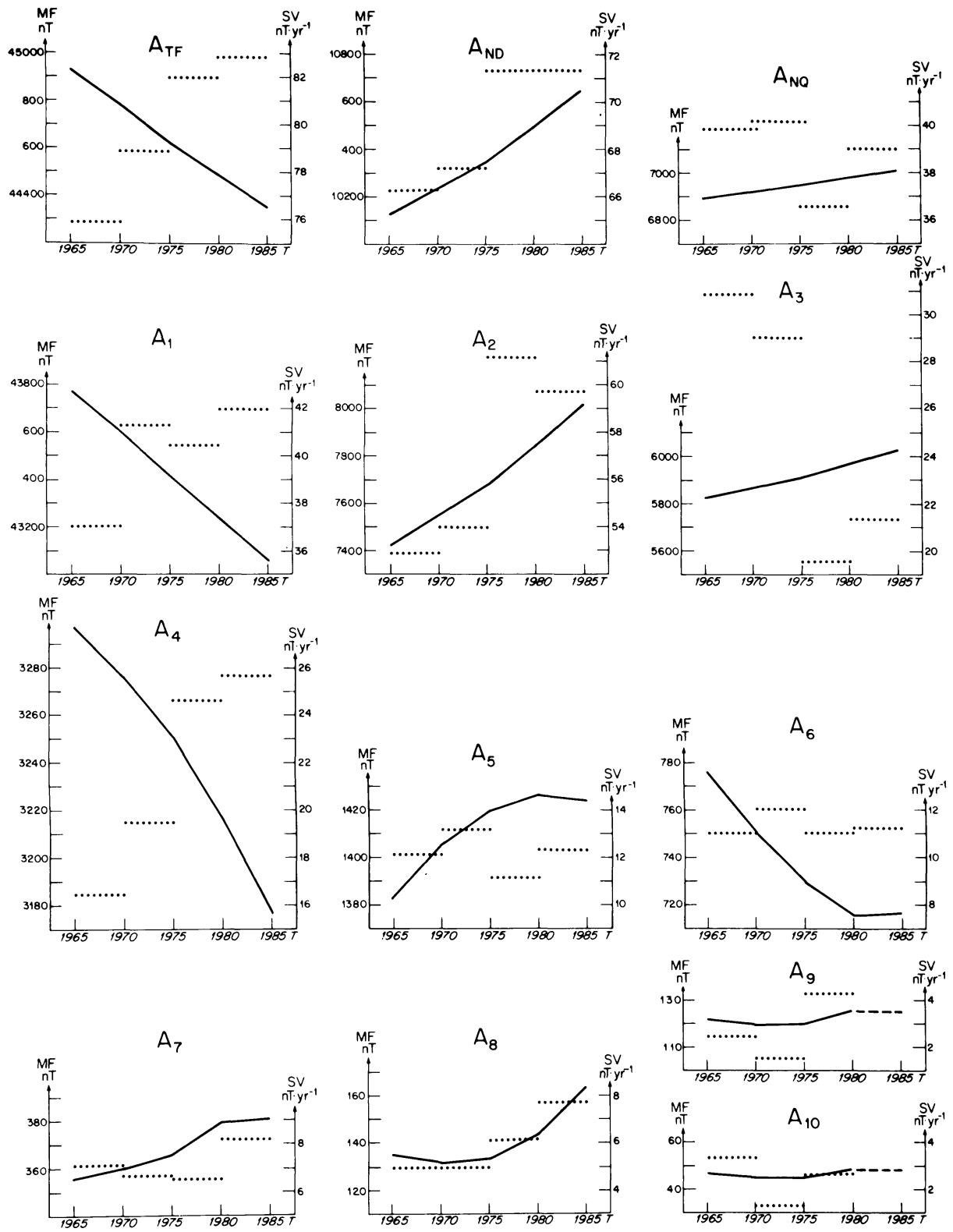

Fig. 1. Amplitudes of the total field $\left(A_{\mathrm{TF}}\right)$, non-dipole field $\left(A_{\mathrm{ND}}\right)$, non-quadrupole field $\left(A_{\mathrm{NQ}}\right)$, and of the individual $n$ th-degree harmonic fields $\left(A_{n}\right)$ of the main field (MF) and secular variation (SV) models shown as functions of time. The MF amplitudes are represented by the solid lines, the SV amplitudes are shown as dotted horizontal bars, each covering the five-year period of the validity of the given SV model. Note the difference in the MF scale between the first two and the last two rows of the plots. 
Amplitudes of the secular variation show mainly a quadratic or cubic dependence on time. In spite of this variability the SV amplitudes for both the total and non-dipole fields increase systematically. This is not true for the non-quadrupole field, however, since its SV amplitude has a distinct minimum in the interval 1975-80. The minimum is due to the behaviour of the third harmonic: among the four lower-degree harmonics, dominating the total field secular variation, it is the only one whose SV amplitude shows a significant decrease. It can be also noted that the changes of the SV amplitude of the third harmonic are opposite to the changes for the second-degree harmonic.

The changes of the secular variation amplitudes show an interesting pattern. Excluding the two highest-degree SV harmonics which are not defined for the interval 1980-85, six of the remaining harmonics have an extremum of the SV amplitude in the interval 1975-80. Moreover, five of them have minima, the only exception being the second-degree harmonic. It cannot be answered at present, however, whether this effect represents an actual behaviour of the internal geomagnetic field or whether it is due to the transitions between the definitive SV model for the interval 1970-75, the provisional model for 1975-80 and the predictive model for 1980-85. Another possibility is that it might be connected with the solar activity having its minimum at 1975.

In Fig. 2, the amplitude spectra of the main field and secular variation as well as their ratio are plotted on a semi-logarithmic scale as functions of the degree $n$. To avoid the effects of the discontinuities between the SV models, the values of the SV amplitudes are associated with the middle epochs of the five-year intervals, 1967.5, 1972.5, 1977.5, and 1982.5, and compared with the MF amplitudes at the same epochs.

The most prominent feature of the MF amplitude plots is their almost linear character, corresponding to an exponential dependency of the MF amplitude on the degree $n$. There are two major exceptions at both ends of the plots. The first and the ninth-degree harmonic amplitudes are higher than needed to satisfy an exponential decay, while the amplitudes of the second and the eighth-degree harmonics are lower. In spite of that, the general linearity of the MF spectrum confirms the belief that the transition between the dominance of the core field and that of the crustal field occurs above the tenth degree. However, the change of the slope between the eighth and the ninth harmonics suggests that the transition might occur between these harmonics (NAGATA, 1965).

The spectra for the secular variation are dominated by the second-degree harmonic amplitude. They are much more irregular, especially at the epochs 1977.5 and 1982.5. This is due mainly to the decrease of the SV amplitude of the third-degree harmonic accompanied by the simultaneous increase of the SV amplitude of the fourth-degree harmonic. In spite of these irregularities, a tendency for a linear decay with $n$ can still be observed in SV amplitude spectra, although its rate is lower than for the main field models.

The ratio between SV and MF amplitudes describes relative secular variation of the field. It grows with $n$, changing from about 0.1 per cent per year for the first-degree harmonic to almost $10 \%$ per year for the highest ones. This again confirms that the tenthdegree model contains mainly the core field, since the relative secular variation of the crustal field should be much smaller. There are certain irregularities in the plots - the values of the ratio for the eighth-degree harmonic are relatively larger, while the values for the first-degree harmonic, for the ninth-degree harmonic at the two earlier epochs, and for the third-degree harmonic at the two later epochs are relatively smaller.

To summarize, there are several general conclusions concerning the Earth's magnetic 


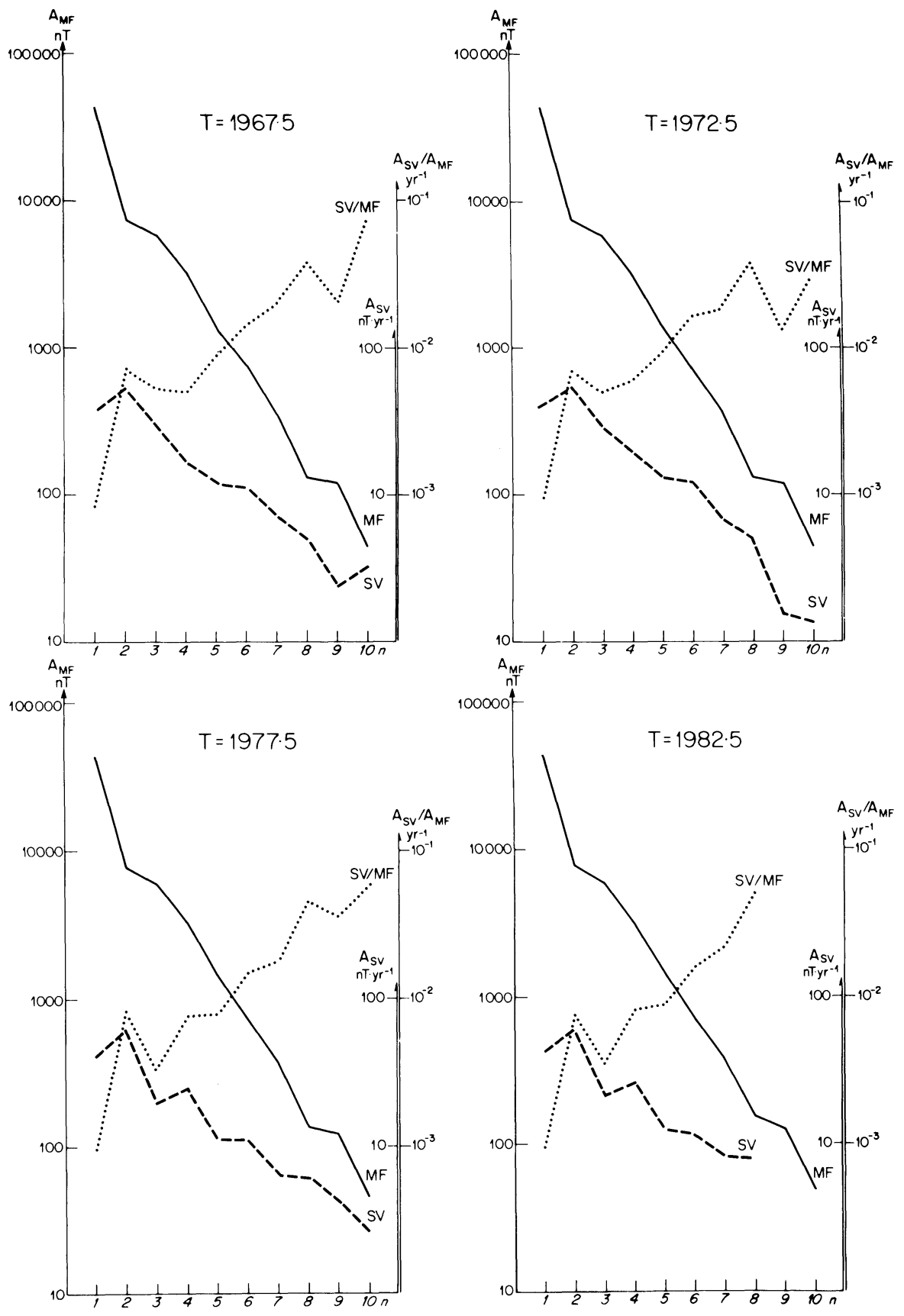

Fig. 2. Amplitude spectra of the main field (MF) and secular variation (SV) models and the ratio of these amplitudes (SV/MF) shown as functions of the degree $n$. The vertical scales are logarithmic. 
field, as described by the set of the International Geomagnetic Reference Field models.

(i) The decay of the total main field and the simultaneous growth of the non-dipole field indicate that there is an energy transfer from the first-degree harmonic to the higherdegree ones.

(ii) The generally linear character of the main field and secular variation spectra confirms the dominance of the core field in the tenth-degree spherical harmonic model.

(iii) The amplitude of the secular variation increases significantly during the period 1965-1980 for both total and non-dipole fields. The increase for the interval 1980-85 is much smaller; this effect may be real or may be due to the uncertainties in the predictive secular variation model for the interval 1980-85.

(iv) There are two distinct groups of SV harmonics. The first group consists of the four lowest-degree harmonics, with their SV amplitudes having high values and changing significantly. The second group consists of the remaining harmonics of degree five to ten. Their SV amplitudes are generally smaller and change less. Different behaviours of these two groups may indicate different origins of the two parts of the field.

(v) The secular variation field shows a very distinctive behaviour in the interval 1975-80: the second-degree SV harmonic has a maximum while five other harmonics have minima in this interval. For definitive conclusions to be drawn regarding the nature of this effect, reliable data spanning over at least three solar cycles are necessary.

The author thanks R. L. Coles, L. R. Newitt, and E. Dawson for their comments.

\section{REFERENCES}

IAGA Division I Working Group 1, International Geomagnetic Reference Fields: DGRF 1965, DGRF 1970, DGRF 1975 and IGRF 1980, EOS Trans. AGU, 62, 1169, 1981.

Nagata, T., Convergency of the Spherical Harmonic Coefficients of the Geomagnetic Field, J. Geomag. Geoelectr., 17, 153-155, 1965.

Ostrowski, J. A., A Comparison of the Proposed IGRF Models: Internal and Relative Consistencies, J. Geomag. Geoelectr., this issue, 401-409, 1982. 